\title{
Computer Simulation of the Impact of Optimization of Width in the Helical Cylindrical Gear on Bearing and Durability. Part 2. Angular Correction of the Gear Profile
}

\author{
Myron Czerniec ${ }^{1}$
}

1 Lublin University of Technology, ul. Nadbystrzycka 36, 20-618 Lublin, Poland, e-mail: m.czerniec@pollub.pl

\begin{abstract}
On the basis of the elaborated calculation method, the optimal width in involute helical gears was determined ensuring a constant length of the line of contact between the meshing gears that is based on the novel method for determining the wear and durability of the gears which was employed to measure the maximum contact pressures, linear wear of teeth and durability of the gear with angular correction of the profile. It was possible to determine the variations in the parameters for the optimized gear describing the meshing gears at different values of the profile correction coefficients.
\end{abstract}

Keywords: block calculation method, cylindrical helical gear, angular correction of engagement, optimal gear width, maximum contact pressures, tooth wear, gear durability

\section{INTRODUCTION}

Cylindrical gears can undergo height correction of the profile which does not cause any change in the distance between their axes or they can be subjected to angular correction of the profile, which does. Besides, the coefficients of profile correction can have different values. Part 1 of this study reported the results of a study investigating a cylindrical helical gear with height correction of the profile. The investigation was conducted using a new method for determining the wear and durability of toothed gears [1]. This paper reports the results obtained for the cylindrical gear with angular correction of the profile depending on the type of engagement [3].

\section{SOLUTION TO THE PROBLEM}

In the helical cylindrical gears with angular correction of the profile, the correction coefficients are $x_{1} \neq x_{2}$ and the total coefficient of profile correction is $x_{\Sigma}=x_{1}+x_{2}$. The real distance $a_{w k}$ between the axes will be higher than the nominal distance $a_{w}$ :

$$
\left.a_{w k}=r_{w 1}+r_{w 2}\right\rangle a_{w}
$$

The rolling pressure angle $\alpha_{w}$ will depend on the real distance between the axes of the meshing gears and will be higher than the apparent pressure angle $\alpha_{t}$. When the real distance between the axes is known

$$
\alpha_{w}=\arccos \frac{a_{w}}{a_{w k}} \cos \alpha_{t}
$$

The radii of the pinion and gear:

$$
r_{w 2}=r_{2} \frac{\cos \alpha_{t}}{\cos \alpha_{w}}
$$

The radius of the addendum:

$$
\begin{aligned}
& r_{a 1}=r_{1}+\left(1+x_{1}-K\right) m, \\
& r_{a 2}=r_{2}+\left(1+x_{2}-K\right) m,
\end{aligned}
$$

where is the coefficient of addendum reduction.

The equations wherein the above parameters of profile correction must be taken into consideration [2]:

$$
N=9550 P K_{g} / r_{w 1} n_{1} \cos \alpha_{w}
$$




$$
\begin{gathered}
\operatorname{tg} \alpha_{t 10}=(1+u) \operatorname{tg} \alpha_{w}-\frac{u}{\cos \alpha_{w}} \\
\sqrt{\left(r_{20} / r_{w 2}\right)^{2}-\cos ^{2} \alpha_{w}} \\
\alpha_{t 1 s}=\operatorname{arctg} \sqrt{\left(r_{1 s} / r_{w 1}\right)^{2}-\cos ^{2} \alpha_{w}} \\
\rho_{t 2 j}=r_{w 2} \sqrt{\left(r_{2 j} / r_{w 2}\right)^{2}-\cos ^{2} \alpha_{w}} \\
r_{2 j}=\sqrt{a_{w}^{2}+r_{1 j}^{2}-2 a_{w} r_{1 j} \cos \left(\alpha_{w}-\alpha_{t 1 j}\right)} \\
r_{1 j}=r_{w 1} \cos \alpha_{w} / \cos \alpha_{t 1 j} \\
\operatorname{tg} \alpha_{t 2 s}=\left(1+u^{-1}\right) \operatorname{tg} \alpha_{w}- \\
-\frac{1}{u \cos \alpha_{w}} \sqrt{\left(r_{1 s} / r_{w 1}\right)^{2}-\cos ^{2} \alpha_{w}} \\
\alpha_{t 2 j}=\arccos \left[\left(r_{w 2} / r_{2 j}\right) \cos \alpha_{w}\right] \\
e_{1}=\sqrt{r_{1 s}^{2}-r_{b 1}^{2}}-r_{w 1} \sin \alpha_{w} \\
e_{2}=\sqrt{r_{20}^{2}-r_{b 2}^{2}}-r_{w 2} \sin \alpha_{w}
\end{gathered}
$$

\section{NUMERICAL SOLUTION}

The input data included: $z_{1}=20 ; z_{2} 80$; $m=3 \mathrm{~mm} ; u=4 ; n_{1}=700$ revolutions per minute; $P=5 \mathrm{~kW} ; f=0.05 ; \beta=10^{\circ} ; K_{g}=1.6$. The following materials were used: the pinion was made of 38HMJA steel after nitriding with 58 HRC; $R_{\mathrm{m}}=1040 \mathrm{MPa}, C_{1}=3.5 \cdot 10^{6} \mathrm{~m}_{1}=2$; the gear was made of $40 \mathrm{H}$ steel after bulk heat treatment with $53 \mathrm{HRC}, R_{\mathrm{m}}=981 \mathrm{MPa}, C_{2}=0.17 \cdot 10^{6}, \mathrm{~m}_{2}=2,5$; $E=2.1 \cdot 10^{5} \cdot \mathrm{MPa}$. Lubrication involved the use of an oil characterized by the kinematic viscosity $v_{+50^{\circ}} \approx 15 \mathrm{cSt} ; h_{k^{*}}=0.5 \mathrm{~mm} ; \Delta \varphi=4^{0}$. The profile correction coefficients and the geometrical parameters of the gear were as follows: $x_{1}=0 \ldots 0.5, x_{2}=0 \ldots 0.584 ; x_{\Sigma} 0.584$; $a_{w}=152.314 \mathrm{~mm} ; a_{w k}=154 \mathrm{~mm} ; \alpha_{t}=20.283^{\circ}$; $\alpha_{w}=21.918^{\circ}$. The width of the gear wheels: $b_{w}=54.275 \mathrm{~mm}$ while $l_{\min }=$ const and $\varepsilon_{\beta}=1$; $b_{w}=30 \mathrm{~mm}$ while $I_{\min } \neq$ const and $\varepsilon_{\beta}<1$. The gear with $b_{w}=54.275 \mathrm{~mm}$ is described by tripledouble-triple engagement, while the gear with $b_{w}=30 \mathrm{~mm}$ by double-single-double engagement. The calculations were performed using a block calculation method from the quantity of the interaction block $\mathrm{B}=2100000 \mathrm{rpm}$.
The numerical results are given in the figures below. Figure 1a shows the initial maximum contact pressures $p_{\text {jmax }}$ which occur during triple-double-triple engagement while Figure $1 \mathrm{~b}$ illustrates the variations in due to the wear of gear teeth amounting to $0.5 \mathrm{~mm}$.

The highest contact pressures $p_{\text {imax }}$ can be observed at the start of double engagement. Later on, when the meshing gears are in triple engagement, the pressures $p_{\text {imax }}$ decrease by up to 1.7 times. In other cases, the decrease in the pressures is smaller and depends on the values of the profile correction coefficients.

Figures 2 and 3 illustrate the linear wear of the gear teeth $h_{2 j}$ and the pinion $h_{1 j}$.

The maximum allowable wear is first reached by the gear teeth at different characteristic points of pressure depending on the values of the profile correction coefficients - at the start of triple engagement and almost at the start of double engagement (Fig. a) or at the end of double engagement (Fig. b). Similar observations can be made with the maximum allowable wear of the pinion teeth.

The relationship between the minimum durability of the gear and profile correction is shown in Figure 4.

The highest durability can be observed for the gear with profile correction when $x_{1} \approx 0.2$, $X_{2} \approx 0.384$; this value is higher by 1.40 than that of the gear without profile correction.

In order to compare the effect of the gear width on the type of engagement, the results of the contact, wear and durability of two gears are as follows: the gear described by the width $r$ and double-single-double engagement and the gear described by the width $b_{w}=54.275 \mathrm{~mm}$ and triple-double-triple engagement. Figure 5 presents the results of the maximum contact pressures $p_{\text {jmax }}$ for the two tested gear widths.

Therefore, increasing the gear width by 1.81 times leads to an almost proportionate decrease (by $1.86 \ldots 1.8$ times) in the initial contact pressures at the start of engagement and by $1.89 \ldots 1.92$ times at the points of change of the type of engagement. It must be emphasized that the double engagement zone of the gear with the width $b_{w}=54.275 \mathrm{~mm}$ is $3-4$ times higher than in the case of narrower gears, which is vital from a practical point of view.

As it can be observed in Figure 6, the gear width and the type of engagement have a greater effect on the minimum durability of the gear. 

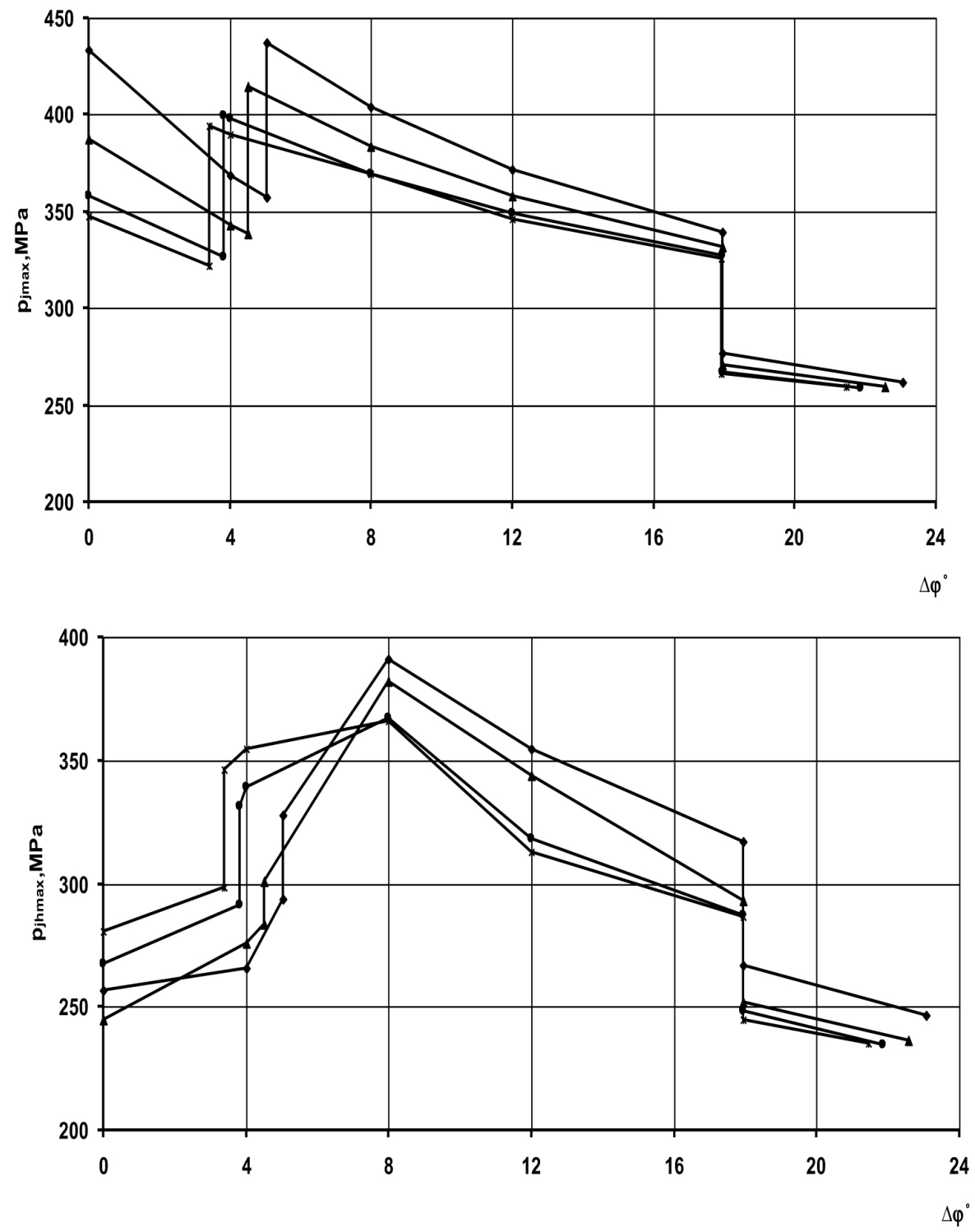

\begin{tabular}{|c|c|}
\hline$\leftarrow \times 1=0, \times 2=0.584$ & 士 $\times 1=0.2 \times 2=0.384$ \\
\hline$\rightarrow \times 1=0.4, x 2=0.184$ & \# $\mathrm{x} 1=0.5, \mathrm{x} 2=0.084$ \\
\hline
\end{tabular}

Fig. 1. Variations in the pressures $p_{j \max }$ during the meshing of the teeth due to their wear
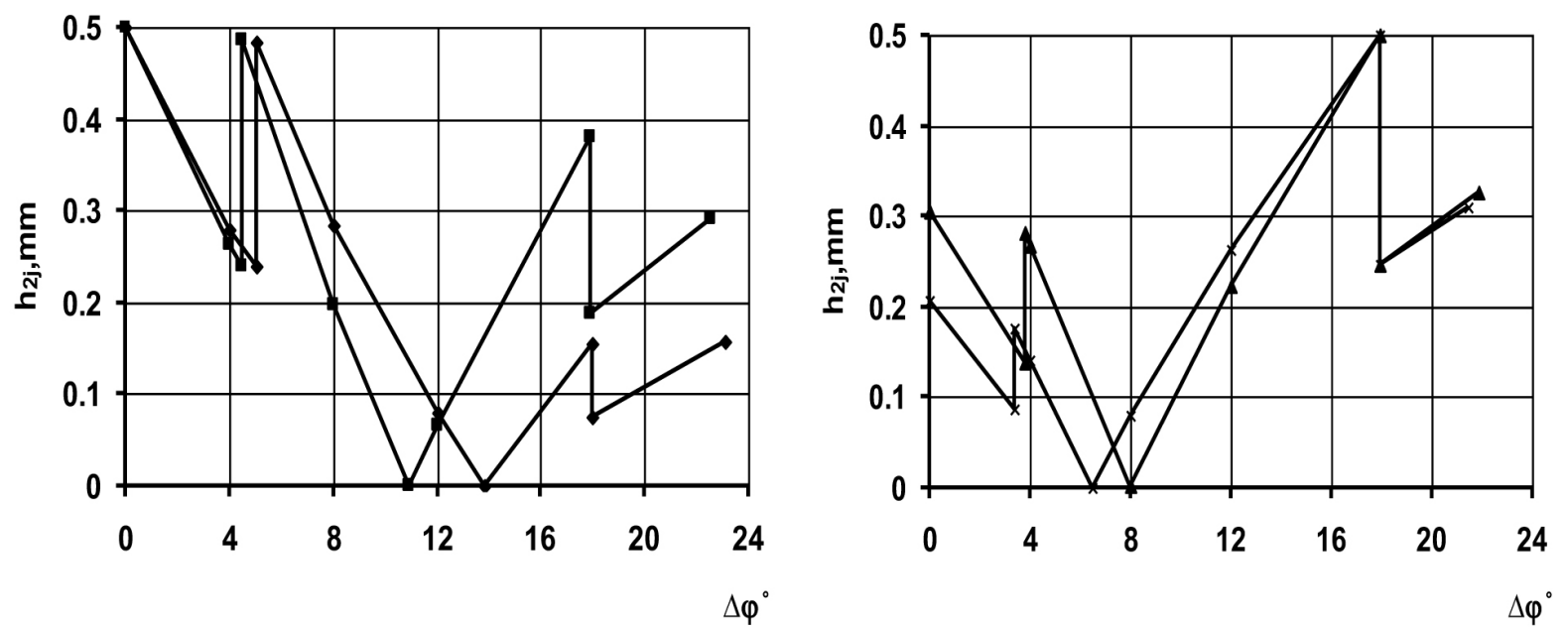

Fig. 2. Linear wear of the gear teeth along their profile 

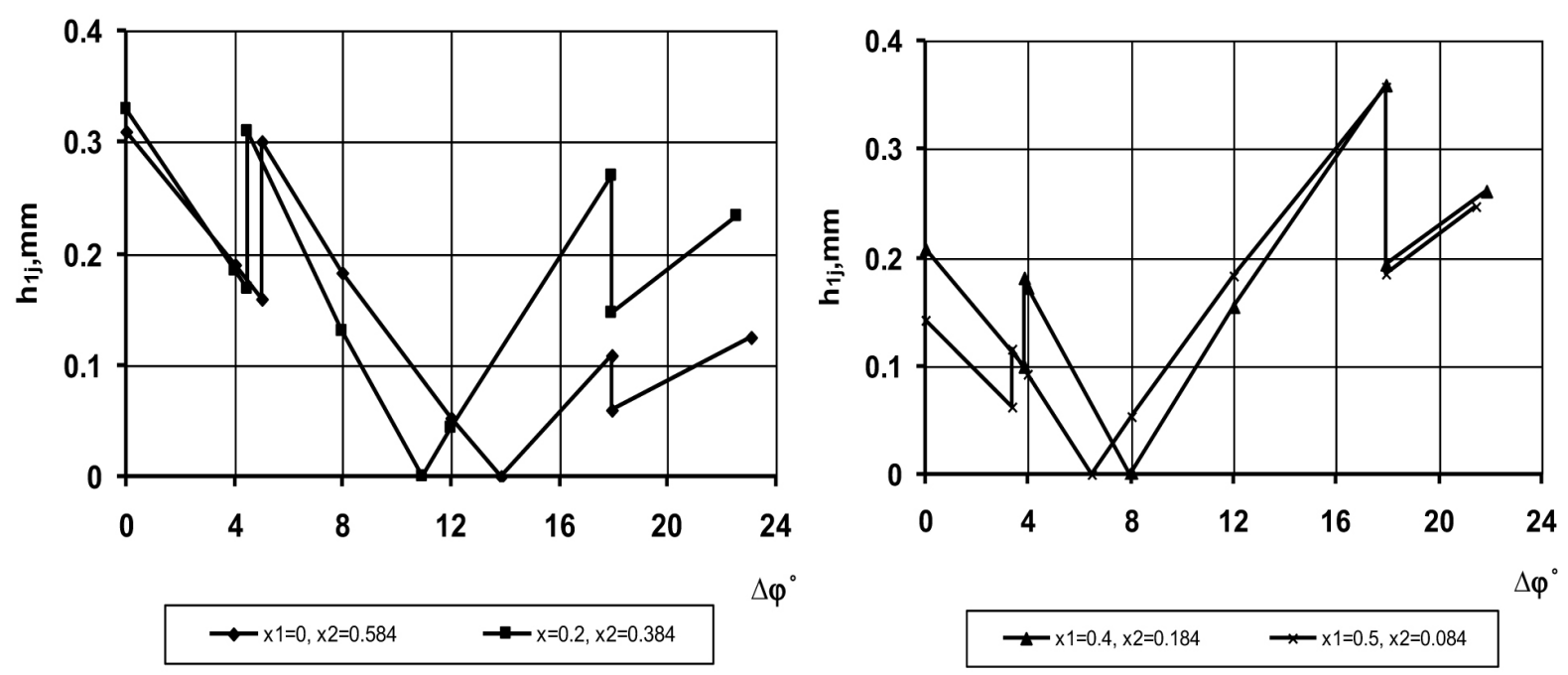

Fig. 3. Linear wear of the pinion teeth along their profile

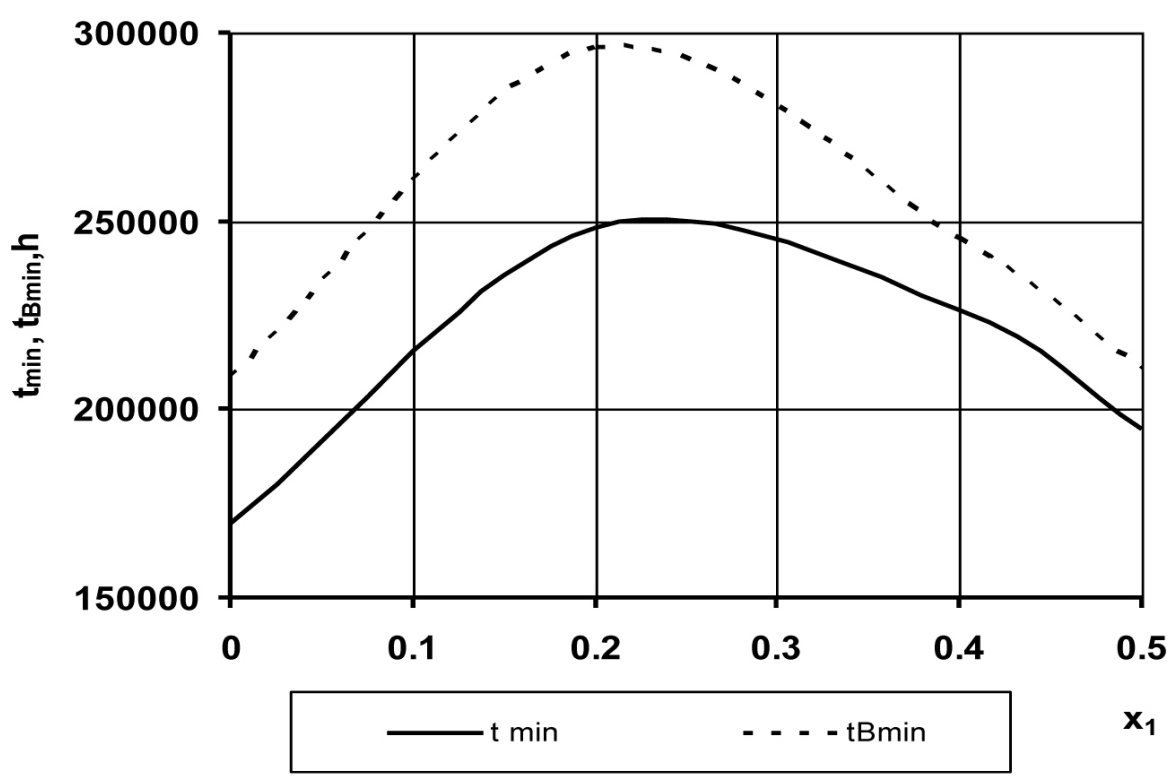

Fig. 4. Minimum durability of the gear versus profile correction: solid line $\mathrm{t}_{\text {min }}$ when $p_{j \max }=$ const, dotted line $-t_{B \text { min }}$ when $p_{j h \max }=v a r$

For the gear with the profile correction coefficients $x_{1} \approx 0, x_{2} \approx 0.584$, the durability increases by 9.2 times, and when the optimum profile correction coefficients are applied $x_{1} \approx 0.2, x_{2} \approx$ 0.384 - by 8.8 times.

A similar effect of gear width can also be observed in the case of the gear with profile correction, as described in Part 1 of the paper. Therefore, it can be concluded that the two tested types of profile correction exert a similar impact on the contact and tribotechnical parameters of the gear.

\section{CONCLUSIONS}

1. A numerical problem concerning the setting of maximum contact pressures, linear wear and durability of cylindrical gears was solved using an original method for determining the wear and durability of gears which takes into account the profile correction and type of engagement.

2. Two types of gear were examined: a gear with optimized width of the gear wheels ensuring a constant force between the meshing teeth and a gear with a smaller width of the gear wheels. 

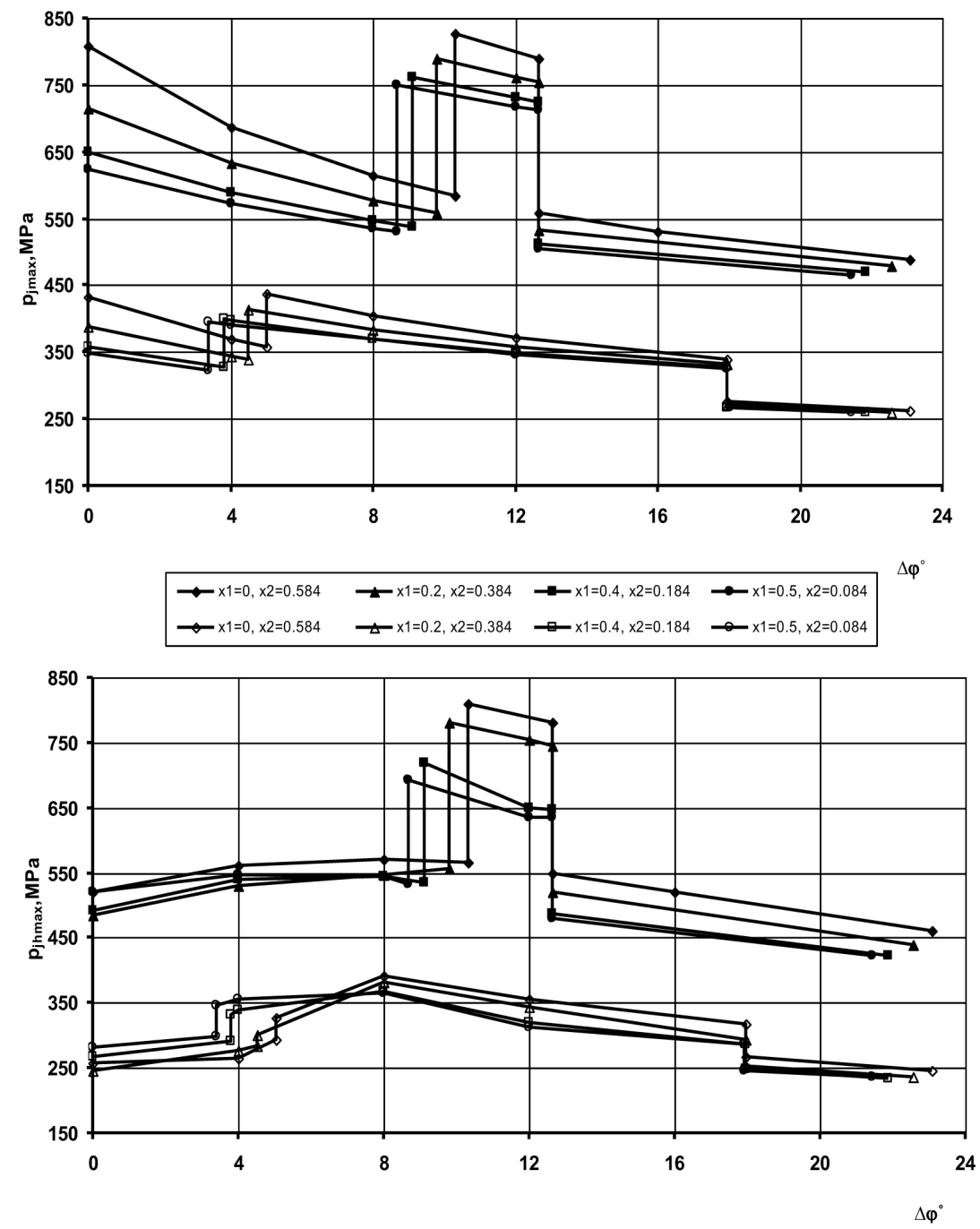

Fig. 5. Maximum contact pressures (a) and their variations (b) due to wear of the teeth: $b_{w}=30 \mathrm{~mm}$ (top), $b_{w}=54.275 \mathrm{~mm}$ (bottom)

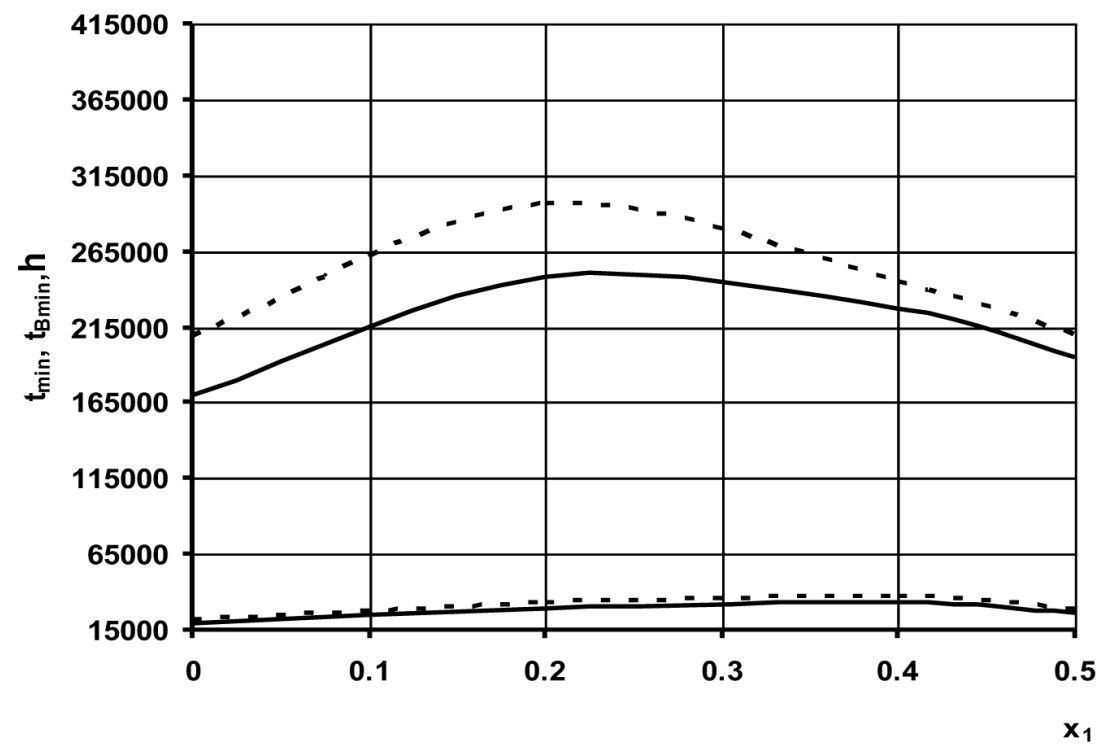

Fig. 6. Impact of profile correction on minimum durability of the gear at $b_{w}=54.275 \mathrm{~mm}$ (top), $b_{w}=30 \mathrm{~mm}$ (bottom) 
3. It was found that the increase in the gear width ranging from 30 to $54.275 \mathrm{~mm}$ results in an almost proportionate decrease in the maximum contact pressures.

4. The increase in the gear width by 1.81 times leads to a considerable increase in the minimum durability of the gear - by 9.2-8.8 times, depending on the value of the profile correction coefficient.

5. Regardless of the type of applied profile correction (angular or height correction of the gear profile), the width of the gear wheels has the greatest impact on the contact strength and wear of the gear teeth.

6 . The application of suitable values of the correction coefficients of the pinion and the gear lead to a uniform wear of the gear teeth for both engagement zones.

\section{REFERENCES}

1. Chernets M.V., Yarema R.Ya., Chernets Yu.M., A method for the evaluation of the influence of correction and wear of the teeth of a cylindrical gear on its durability and strength. Part 1. Service live and wear. Materials Science, 2012, 3, 289-300.

2. Chernets M., Kiełbiński J., Chernets Yu., A study on the impact of teeth meshing conditions and profile correction on the carrying capacity, wear and life of a cylindrical gear. Tribologia, 2016, $2,25-43$.

3. Chernets M., Computer Simulation of the Impact of Optimization of Width in the Helical Cylindrical Gear on Bearing and Durability. Part 1. Height Correction of the Gear Profile. Adv. Sci. Technol. Res. J. 2019, 13(1), 52-59. 